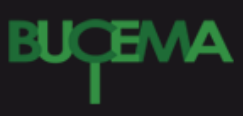

Bulletin du centre d'études médiévales d'Auxerre | BUCEMA

Hors-série $n^{\circ} 7 \mid 2013$

Les nouveaux horizons de l'ecclésiologie : du discours clérical à la science du social

\title{
L'Église au miroir. Visiter le musée ecclésial avec Pierre Legendre
}

\section{Alain Rauwel}

\section{(2) OpenEdition}

\section{Journals}

Édition électronique

URL : https://journals.openedition.org/cem/13156

DOI : $10.4000 /$ cem. 13156

ISSN : 1954-3093

Éditeur

Centre d'études médiévales Saint-Germain d'Auxerre

Référence électronique

Alain Rauwel, "L'Église au miroir. Visiter le musée ecclésial avec Pierre Legendre », Bulletin du centre d'études médiévales d'Auxerre | BUCEMA [En ligne], Hors-série n 7 | 2013, mis en ligne le 06 août 2013, consulté le 04 mars 2023. URL : http://journals.openedition.org/cem/13156 ; DOI : https://doi.org/ 10.4000/cem. 13156

Ce document a été généré automatiquement le 4 mars 2023.

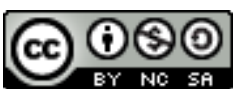

Creative Commons - Attribution - Pas d'Utilisation Commerciale - Partage dans les Mêmes Conditions 4.0 International - CC BY-NC-SA 4.0

https://creativecommons.org/licenses/by-nc-sa/4.0/ 


\title{
L’Église au miroir. Visiter le musée ecclésial avec Pierre Legendre
}

\author{
Alain Rauwel
}

1 Pierre Legendre qui, nonobstant son intérêt profond pour l'Afrique, ne cultive pas une affection débordante à l'égard de l'anthropologie structurale, conseillerait volontiers, à qui voudrait s'enquérir de ce qui nous fait les étranges choses que nous sommes, de visiter Notre-Dame plutôt que le quai Branly: «l'Église occidentale (...) transporte encore sous nos yeux son musée vivant, un réservoir de mythes fondamentaux qu'on pourrait croire à jamais perdus et dont l'anthropologie devrait s'occuper davantage » (AC $27^{1}$ ). Le Palatin, en effet, est en ruine tandis que, malgré tous les aggiornamenti, de l'autre côté du Tibre parle encore un pontife «qui a tout le droit dans l'archive de sa poitrine» (AC 75$)$ et dont les ministres accomplissent partout les rites propres à constituer l'humanité en société sacrée. Si donc l'Église n'est jamais vraiment l'objet unique et principal de l'interrogation de Legendre, elle est partout dans son œuvre, sa tradition textuelle et liturgique irriguant tous les grands thèmes des Leçons en même temps qu'elle reçoit de cette glose aussi infinie que celle des maîtres médiévaux une lumière qui, pour être souvent indirecte, n'en est pas moins crue.

\section{Texte, Image, montages}

2 Toute institution repose sur un nom, un au nom de, sur lequel s'appuie tout l'édifice normatif. Legendre désigne cette pierre angulaire comme « la Référence absolue ». Bien entendu, rien n'oblige à ce que cette Référence soit autre chose qu'un mot; nul ne va jamais, soulevant le voile du Temple, voir ce que désigne la parole ultime, il suffit que cette parole soit prononcée -mais surtout pas par n'importe qui, ni n'importe comment: le Pontife, prêtre et docteur, a le monopole de la profération de la Référence, à la fois en termes de légitimité et d'efficience. La structure ici observée du point de vue du droit consonne parfaitement avec toute la métaphysique de Chrétienté, qui a été très justement définie comme « métaphysique de l'Exode » en référence à Ex 3 , $14:$ « je suis Celui qui est ». L'Être autour duquel s'est empressée la théologie médiévale 
n'est pas tant épiphaniquement révélé que désigné, et auto-désigné. C'est à partir de son nom, indiscutable puisque venant d'En-haut, que toute pensée devient possible, comme tout ordre ecclésial. Ainsi, si le lieu de la Référence absolue est une place vide, sa fonction est néanmoins occupée: par le Texte. Au-delà du nom initial se fait entendre, solennel, un «discours inaugural » en forme de sociogonie. Sans lui, il ne saurait y avoir société, car la société, d'un certain point de vue, n'est rien d'autre que le discours sacré qui la fonde (De la Société comme Texte, 2001), proféré du ton auquel on n'a recours qu'au temple ou sur scène. " Nous sommes dans le théatre occidental des textes et nous n'en sortirons pas » (L VII 210), avertit Legendre.

3 Selon lui, l'une des grandes caractéristiques du système d'Occident est de ne pas absolutiser le texte comme un indépassable, incommentable, intraduisible, ce que fait à ses yeux la tradition juive (L VII 296) ${ }^{2}$, mais de le rendre accessible seulement par la médiation d'une interprétation, donc d'un interprète. "L'institution, qui est-ce, sinon aussi le décrypteur qualifié de la Loi, dont le commentaire finit par faire corps avec le texte? » (AC 80) L'interprète est « souverain », car il est indispensable à la construction de la communauté humaine: sans lui, pas de «discours inaugural», donc pas de société. Legendre, qui a du goût, on le reverra, pour les qualifications révolutionnaires, désigne alors l'occident latin comme le lieu d'une "révolution de l'interprète». Conception posant la nécessité d'un Tiers, et comme telle éminemment catholique, au sens moderne du terme, c'est-à-dire étrangère aux mythologies de l'accès direct au Verbe, de l'âme seule face à son créateur, de l'épouse (fidèle ou Église) introduite dans la chambre secrète de l'Époux. Chez Legendre, si noces il doit y avoir, un terzo incommodo tient la chandelle. Plaisamment, il donne parfois un visage à cet interprète si nécessaire. Au début des Leçons III, Dieu au miroir, une dédicace présente le livre à Guillaume Durand de Mende, un juriste, rubriciste et prélat du XIII ${ }^{e}$ siècle. Si Durand est plutôt connu des historiens pour son Rational des divins offices, la plus grande somme liturgique du Moyen Âge, il l'est des juristes pour son Miroir du droit, qui lui a valu le surnom de "Spéculateur ». Aucun hasard, bien sûr, dans la promotion de Durand le Spéculateur: il s'agit bien de faire entendre que le discours de l'interprète est en somme le stade du miroir des sociétés chrétiennes ${ }^{3}$. Elles se reconnaissent comme sociétés dans le miroir que leur tend l'interprète. Et rien, du coup, n'est plus logique que de voir dans la psychanalyse une « seconde révolution de l'interprète ».

Ce n'est d'ailleurs pas au Texte seul que s'adossent les discours d'instauration. Le miroir dans lequel la société se dévisage reflète, par nature, des images. Pierre Legendre est extrêmement sensible à la dimension iconique de la culture romano-canonique. Il aime à inclure dans ses livres des commentaires d'œuvres peintes ou gravées. C'est que l'image n'est pas seulement une production humaine, aussi riche soit-elle, elle est l'homme lui-même en tant qu'il est image de son créateur, imago Dei. Comme l'autodéfinition de Yahvé devant Moïse est la clef de la métaphysique chrétienne, le « créé à l'image et à la ressemblance de Dieu " de la Genèse est celle de l'anthropologie médiévale, à la fois méditation d'un principe fondamental et exploitation résolue des potentialités de sa double dénomination ${ }^{4}$. Si en effet la ressemblance est ce qui est perdu par le péché, que demeure-t-il en la créature qui porte le sceau du créateur? L'image, bien sûr, comme trace ineffaçable de la première gloire de l'homme : l'homme est «sujet institué » en tant qu'il est «image vivante de l'Image absolue » (L III 17). Dans la regio dissimilitudinis, la théorie de l'image fait revenir aux sens spirituels comme une fragrance du jardin perdu. Il est bien certain que c'est avec en tête cet univers de 
pensée que Legendre aborde avec gourmandise les conjonctions juridico-théologiques du texte et de l'image, ce qu'il qualifie de « montages »-montages psychiques aussi bien que doctrinaux, qui ne sont pas sans rappeler la double imago du premier Lacan : celle du corps propre d'une part (via le miroir), et l'imago familiale d'autre part ${ }^{5}$.

5 Les montages les plus décisifs sont de nature rituelle; ce sont les «techniques de célébration », qui ont pour objet de "faire face au vide structural, c'est-à-dire de le rendre pensable en le parlant pour le compte de la société » (L VII 30). C'est l'une des intuitions les plus fécondes de Legendre que d'avoir souligné, à la suite des premiers maîtres de l'ethnologie, combien les sociétés, les latines comme les autres, se disent par leurs rites, combien en conséquence l'exégèse de ces derniers est un lieu décisif de compréhension des mécanismes qui les régissent. Les liturgies ne sont pas la répétition mécanique d'un ordre vain, elles constituent un espace d'intelligibilité maximale, au point que les choix existentiels les plus radicaux, le monachisme en régime de Chrétienté, aboutissent à une "ritualisation intégrale de la vie " (L VII 347). Et leur forme contraignante saisit l'acte même par lequel elles sont analysées: «le commentaire (l'acte de l'interprète médiéval) est d'abord un rituel » (AC 92). L'intérêt actuel pour l'iconographie du docteur donne pleinement raison à Legendre : que l'on pense aux tombeaux solennels des docteurs de Bologne, exposés au cœur de la cité ${ }^{6}$, ou à la représentation très codifiée du maitre enseignant en tête des traités manuscrits. Les procédés stéréotypés, éminemment formulaires, par lesquels s'exprime la glose, participent aussi de cette ritualisation du discours de l'interprète, par lequel il acquiert sa vérité décisive -selon la célèbre formule foucaldienne sur le vrai : "un discours prononcé par qui de droit et selon le rituel requis ${ }^{7}$ ".

\section{La « révolution grégorienne »}

6 En cherchant à préciser la chronologie des grands mouvements précédemment indiqués, Pierre Legendre met fortement en lumière le tournant que constitue la deuxième moitié du $\mathrm{xl}^{\mathrm{e}}$ siècle, ce qu'il n'hésite pas à nommer, au-delà du vocabulaire très convenu de la réforme, la "révolution grégorienne ${ }^{8}$ ». Ce choix nous étonne moins, en 2013. Il ne faut pourtant pas oublier que la médiévistique a vécu pendant des lustres entre une historiographie allemande de la crise grégorienne qui axait sa présentation sur des faits politiques lourds de conséquence, sans doute, mais pas déterminants en dernière instance, et une historiographie romano-française, à la Fliche, qui avait tendance à prendre pour argent comptant les propos exaltés des purificateurs du sanctuaire. L'essentiel, à savoir le coup d'État monastique aboutissant à la réduction de l'Église-peuple à une Église-clergé, demeurait en tout cela peu visible. Legendre, dès ses premiers travaux, a contribué à décrire plus justement le coup de gouvernail le plus radical qui ait jamais changé la course du navire ecclésial -même s'il a probablement, ce faisant, durci l'opposition entre Rome et Byzance, voyant « deux religions distinctes " (L III 290), en grande partie à cause de rapports à l'image différents, là où l'on est bien plus sensible désormais aux continuités, aux échanges, derrière le rideau de fumée d'un « schisme » qui, au moins jusqu'au XIII ${ }^{e}$ siècle, n'en est pas vraiment un.

7 Chez lui, Grégoire VII qui, par une commodité discutable mais universellement partagée, incarne ce moment, est désigné de manière quasi nietzschéenne comme « figure du destin européen » (L VII 107). En effet, il marque symboliquement la fin d'un 
système, celui d'«une économie juridique fondée sur des textes reçus comme immémoriaux et transmis comme indépassables " (L VII 109). Désormais, le pontifelégislateur, en tant qu' «Interprète omniscient", acquiert le statut de "maître des écrits ». On commence à lui appliquer toutes les formules révélées et étudiées par L'Amour du censeur, que résume l'idée de Lex animata. Sur ces bases naît la monarchie pontificale, soit l'assimilation pleine et entière par la tête de l'Église romaine des pratiques et des discours impériaux païens, assimilation certes bien plus ancienne, mais auparavant fort imparfaite. Le résultat est, en son genre, un véritable totalitarisme, appuyé sur une propagande effrénée. Legendre a raison de voir dans l'appel permanent à la "réforme", contre tous les épigones de Fliche, un authentique "slogan" (L VII 108). Du point de vue du droit, c'est alors que la fameuse formule de «système romano-canonique » prend tout son sens.

\section{Du droit à la théologie : exégèse des sacrements}

8 Mais le juriste Legendre ne demeure pas dans le champ clos de sa discipline. Il en repère les prolongements partout dans l'édifice doctrinal, et singulièrement dans un champ décisif pour la compréhension de la structuration de l'Occident latin en Chrétienté : celui de la théologie des sacrements. Les historiens de l'Ecclesia médiévale s'accordent aujourd'hui pour reconnaître que l'un des ingrédients les plus décisifs de l'explosion grégorienne a été la querelle eucharistique issue des thèses de Bérenger de Tours. Le long affrontement entre tenants du vieux symbolisme augustinien (Bérenger) et partisans acharnés du nouvel hyper-réalisme (le parti romain) a mis la question du sacrement de l'autel au cœur de la réflexion sur l'appartenance ecclésiale. Plusieurs générations de maîtres, tout au long $\mathrm{du} \mathrm{XII}^{\mathrm{e}}$ siècle, ont scruté ce problème et tenté de préciser l'appareil conceptuel et lexical nécessaire à son règlement, construisant progressivement la sacramentaire scolastique. En définissant qui accédait légitimement à la table eucharistique, ils disaient en même temps le dedans et le dehors de l'Églisesociété. Pierre Legendre a parfaitement senti l'importance de ce corpus de textes. Audelà de leur dimension très technique, il a repéré en eux, à la suite du P. de Lubac ${ }^{9}$, un lieu capital pour la pensée de la communauté et du corps social, comme le montre le « détour par la théorie des sacrements » introduit dans les Leçons III ${ }^{10}$.

9 Le mot « sacrement » couvre en effet un spectre bien plus large que ne le laisse deviner son sens scolaire défini depuis le XIII ${ }^{\mathrm{e}}$ siècle. En latin classique, le sacramentum est un serment, notamment militaire. Ce qui est en jeu est donc la création de fidélités et de réseaux entre membres d'une société. À l'âge patristique, ce sens premier s'enrichit de toutes les harmoniques de la pensée chrétienne du mystère, en tant que mysterion / mysterium désigne de manière indissoluble à la fois les actes salutaires accomplis par le Christ, leur réitération liturgique et la grâce qu'ils procurent. La sacramentalité est ainsi participation à la grâce capitale, créatrice de communauté dans la mesure où elle résulte de l'approche commune d'une source unique. Elle est aussi productrice de sens, et même creuset de toute signification. Comme l'a très bien vu Legendre, c'est tout l'arsenal conceptuel rendu nécessaire par l'expression adéquate de la co-présence du Christ au ciel et dans l'hostie qui a fourni au monde latin les instruments de sa sémiologie, largement fondée sur des binômes : veritas / figura, signum/res, etc. De ce point de vue, les Leçons ont contribué à poser les jalons d'une réflexion qui aboutit aux travaux d'Irène Rosier établissant la matrice sacramentelle des théories du signe ${ }^{11}$. 
10 Fille de la latinité, la sacramentaire est aussi le révélateur de la nouveauté du régime chrétien de la vérité. Les scolastiques ont cherché à la mesurer en s'interrogeant sur la différence entre les sacrements d'institution dominicale et ce qu'ils appelaient «les sacrements de l'Ancienne Loi ", comme la circoncision: ces derniers sauvaient-ils ? Conféraient-ils une grâce? Laquelle? L'histoire moderne des doctrines a fait peu de place à cette question, qui n'a pas trouvé son explorateur ${ }^{12}$ : il n'en est que plus remarquable qu'elle soit apparue à Pierre Legendre comme "fondamentale» (L III 206). Mieux connue désormais est l'émergence de la figure sacerdotale comme ministre des sacrements. Legendre a repéré le paradoxe autour duquel elle se construit : seul à l'autel, maitre du rite, un homme dit ego. Mais cet ego n'est pas le sien, puisqu'il parle in persona ipsius Christi; il tient le rôle (car telle est bien, dans sa dimension théâtrale, l'acception de persona) de Dieu. Sa parole est transsubstantiatrice, à ceci près que ce n'est pas lui qui parle, mais bien le Verbe par lui. Rien de plus singulier que ce mélange d'autorité et de dépossession, que cette sainte schizophrénie ; pas de sujet plus étrange que celui qui doit se dissoudre comme individu pour accéder à la figure du Chef. Legendre sent bien qu'une affaire décisive se joue ici, y compris dans ses implications théologico-politiques. Il le sent d'autant mieux que l'un des premiers mots du Digeste lui inspire une méditation infinie, quasi bachelardienne : «on appelle les juristes des prêtres »...

11 On suivra moins facilement l'auteur des Leçons lorsqu'il entend nous convaincre que le premier des sacrements, celui dont l'intelligibilité gouverne tout le reste, l'Eucharistie, relève d'une analyse psychanalytique en termes de scène primitive, et doit donc s'inscrire dans la grande problématique legendrienne, celle de la généalogie et de la filiation ${ }^{13}$ : « le montage eucharistique est un montage de filiation » (L III 215). Ce n'est pas ici le lieu d'affronter directement ce problème, dans la mesure où il est à la fois le plus glosé de tous ceux que Legendre a abordés, et le plus fécond chez les épigones en prolongements aussi variés que discutables ${ }^{14}$. On ne peut que constater comment, grâce à un dictum de Gratien certes très riche mais non dominant dans la tradition théologique, la sacramentaire est pour ainsi dire rabattue sur la généalogie, presque de force. On comprend bien pourquoi, en une œuvre qui interroge la normativité occidentale, laquelle est toujours fondée sur une remontée vers le Père-Principe. Reste l'ambigüité entre la dimension descriptive du recours à la généalogie, à laquelle on est tout disposé à se rallier, et la dimension prescriptive qui semble parfois en découler, et qui relève de tout autres procédures. À ce point, on préfère, en historien, suivre l'invitation de François Noudelmann à remettre en cause le paradigme généalogique par une "généalogie de la généalogie ${ }^{15}$ ». Car l'enjeu est, au bout du compte, éthique : que produit-on par l'invocation systématique de la filiation? L'avertissement de Foucault garde ici toute sa portée : «l'histoire, généalogiquement dirigée, n'a pas pour fin de retrouver les racines de notre identité, mais de s'acharner au contraire à la dissiper; elle n'entreprend pas de repérer le foyer unique d'où nous venons, cette première patrie où les métaphysiciens nous promettent que nous ferons retour; elle entreprend de faire apparaître toutes les discontinuités qui nous traversent ${ }^{16}{ }^{\prime}$.

À propos d'une autre dimension de la sacramentalité, la référence généalogique est bien plus opératoire : il s'agit de la pénitence, du pouvoir de lier et de délier. Legendre retrouve ici Foucault dans une réflexion sur l'aveu ${ }^{17}$ qui occupe une grande place dans L'Amour du censeur. "Le canoniste inventeur de la confession, voilà le sorcier d'Occident. » (AC 119) Il analyse « la science formidable des confesseurs » (AC 159), leur 
jeu subtil entre confiance et terreur pour susciter le discours de la faute, pour faire prononcer l'accusation non par un tiers purement extérieur, mais par un tiers caché, si bien intériorisé qu'il occupe la conscience et emprunte la voix du pécheur lui-même. Encore une sainte schizophrénie, en somme, et aussi fondamentale que l'autre, puisque le but n'est rien moins que d'" assurer à l'institution d'être investie d'amour par les sujets » (AC 160). Du coup, la pénitence est-elle vraiment fondée sur « la répression de la jouissance ", quand se dire coupable devient source de jouissance ? Plus tard, Pierre Legendre a vigoureusement synthétisé tout ce qui est en jeu à ses yeux dans l'affirmation post-christique de l'Interdit : « l'Interdit est la construction normative qui consiste à lier et délier le sujet: le lier par ligature généalogique au système normatif pour le délier de l'inceste et du meurtre, c'est-à-dire l'inscrire à sa place instituée parmi ses semblables » (L III 39). D'une certaine façon, cette phrase résume tout le système des Leçons: l'interrogation initiale sur la Loi; l'assimilation du lien sacramentel rituellement opéré à une filiation; la double portée anthropologique (prohibition) et analytique (pulsion) du recours à l'inceste et au meurtre; le retour à ce qui est considéré comme la nécessité première, à savoir l'institution.

«La science du religieux, quelle que soit de celui-ci la forme historique, retrouve son objet fondamental: saisir ce qu'implique instituer la vie, c'est-à-dire comprendre le système de répétition d'un discours inaugural, indéfiniment repris et remodelé, et son principe logique, à savoir la reproduction des filiations ${ }^{18}$." Ainsi s'exprimait Pierre Legendre en 1991, invité à réfléchir à la lumière de l'effondrement du bloc soviétique sur la nature des religions politiques. Sa conférence propose, sur la question qui nous intéresse, une synthèse d'une grande clarté. Comme on l'a vu, tous les aspects de cette synthèse ne sont pas d'une égale utilité pour l'historien du fait ecclésial chrétien. Mais il importe de souligner que plusieurs des chemins par lesquels on a pu sortir d'une " histoire vécue du peuple chrétien ", comme on disait au vieux temps du delumisme, coincée entre l'histoire annalistique de l'institution et la description pseudoethnologique des pratiques "populaires », n'auraient pu être empruntés s'ils n'avaient d'abord été balisés par Legendre. Lui-même ne les a pas tous parcourus en personne sur toute leur longueur, mais il a planté au coin du bois, à leur peu visible point de départ, une pancarte suffisamment incitative pour que d'autres s'y engagent. En ce sens, notre travail aujourd'hui ne serait pas ce qu'il est sans Pierre Legendre, sans cet électrochoc que fut pour beaucoup la découverte de L'Amour du censeur, entretenu tome après tome par le flux des Leçons. Sa lecture a imposé (avec d'autres) la légitimité d'une véritable histoire des discours doctrinaux, différente tant de l'histoire des dogmes à l'usage des séminaires que d'une " histoire des idées » indifférente au social. Elle a suggéré l'intérêt d'enquêtes sur le droit comme affirmation ritualisée de la norme. Elle a mis ou remis sur le devant de la scène toutes les formes d'action liturgique, en les proposant aux chercheurs comme un terrain d'une prodigieuse richesse, et qu'il était urgent de réinvestir autrement, au moment où s'éteignaient les derniers gardiens d'un savoir ecclésiastique désormais aboli. En dépit de certaines apparences, sa fécondité historiographique est donc assurée.

14 Sur le terrain analytique, les choses sont sans doute plus confuses. Si Legendre s'inscrit dans les réseaux savants propres à l'histoire du droit, ce n'est pas par ce biais qu'il est entré dans la bibliothèque des historiens du fait religieux. L'Amour du censeur n'eût-il pas été publié dans la collection de Lacan que son audience eût été bien moindre, dans l'atmosphère intellectuelle des années 1970. Même si ses jugements sur le maître et son école sont sévères (« par des abus théoriques et des pratiques insensées, le lacanisme a 
ruiné la clinique », L III 296, ou les sorties contre les « chefferies lacanistes »), Legendre doit donc être vu, au moins au début de son parcours, comme l'une des figures possibles de la psychanalyse du christianisme telle qu'elle a prospéré sur le terreau fécond de la fascination éprouvée par Lacan lui-même pour « la Chose autour de quoi tourne la nostalgie du désir ${ }^{19}$ ». Fils du sérail, demeuré proche de son frère, Dom MarcFrançois Lacan, moine d'Hautecombe et excellent connaisseur des grands textes médiévaux ${ }^{20}$, durablement séduit par la beauté des cérémonies catholiques et le rayonnement de la papauté (on sait qu'il a tenté vainement d'être reçu en audience au Vatican), l'homme des Séminaires connaissait de l'intérieur cette "vraie religion romaine ", ses puissances et ses latences, pour parler comme Dupront ${ }^{21}$. Plusieurs de ses élèves et auditeurs étaient d'ailleurs bien placés pour le lui rappeler, à commencer par un petit bataillon de Jésuites ${ }^{22}$ : il faudrait ici faire sa place, toute singulière, à Michel de Certeau ${ }^{23}$. Mais Pierre Legendre était autre encore; venant d'ailleurs, institutionnellement et intellectuellement, rétif aux chapelles et à leurs chaisières, il suivait son chemin sans s'identifier aux psychodrames du petit monde analytique. De ce point de vue, et aussi surprenant que cela puisse paraître, il n'est pas sans similitudes, au moins en termes de rapports à Lacan et au lacanisme, avec la figure de Françoise Dolto. Tandis que Legendre relisait les monuments du droit et de la théologie de l'Église grégorienne triomphante, Dolto relisait, tout simplement, les Évangiles -qui relèvent aussi, assurément, du monument. Et tandis que le juriste trouvait, de plus en plus, dans la tradition qu'il étudiait, les preuves de la centralité absolue de la question de la filiation, la grande praticienne proposait, interprétant l'épisode du fils de la veuve de Naïm (Lc 7, 11-16), une autre lecture du projet chrétien. Pour elle, le garçon que l'on conduisait à la tombe et que le Christ rend à la vie périssait d'être englué dans l'amour morbide d'une mère veuve, reportant sur lui un désir sans objet conjugal qui l'empêchait de grandir sur le mode indispensable de la coupure et de la séparation. Jésus, tiers étranger au cercle familial, fait entendre une parole de libération. « Il lui donne, par son appel impératif et public, la stature d'homme libre qu'il lui révèle et l'élan pour sa vie à construire, face à la société ébahie qu'il fait taire. Il éveille cet avorton de cœur à sa virilité de cœur ${ }^{24}$. » Sans doute Dolto assimile-t-elle bien vite, à nos yeux, résurrection à renaissance ${ }^{25}$, et par conséquent Verbe incarné à fonction paternelle, de même qu'elle fait bien vite de la liberté du jeune homme le projet d'une " descendance, d'un destin fécond ${ }^{26}$ ». Reste que le geste messianique qu'elle décrit est fondamentalement un geste d'arrachement -arrachement de l'inceste, et dont affirmation de l'Interdit, mais pas exactement au sens que Pierre Legendre donnerait à ce concept. Dolto et Legendre, bien sûr, ne parlent pas du même lieu. Mais ils parlent de la même chose. Et leurs interprétations, à l'un et à l'autre, laissent le lecteur sur une double question : que s'est-il passé sous le nom de «christianisme »? Mais aussi : que voulons-nous faire, aujourd'hui, du legs de ce passé?

\section{NOTES}

1. Abréviations : $\mathrm{AC}=$ L'Amour du censeur $; \mathrm{L}=$ Leçons. 
2. L'opposition entre Rome et Jérusalem sur ce point fait peut-être bon marché de la fonction rabbinique et du midrash.

3. Faut-il rappeler que, si Lacan n'est pas l'inventeur du stade du miroir, c'est à lui qu'il doit sa popularité ? La notion est présente dès l'avant-guerre, notamment dans le fameux chapitre de l'Encyclopédie française. Elle est reprise dans « Le stade du miroir comme formateur de la fonction $\mathrm{du}$ Je telle qu'elle nous est révélée dans l'expérience psychanalytique » (Écrits).

4. Sur les très nombreuses utilisations de ce thème, p. ex. chez s. Bernard, cf. R. JAVELET, Image et ressemblance au XII ${ }^{e}$ s., Paris, 1967.

5. La fascination de Legendre pour l'affaire Lortie, qui a inspiré toutes les Leçons VIII, trouve sa source dans cette phrase du caporal : « le gouvernement du Québec avait le visage de mon père ». Cf. Digeste 11, 7, 44 (cit. L VII) : "c'est de la tête qu'on fait l'image et c'est par elle qu'on reconnaît ».

6. R. GRANDI, I monumenti dei dottori e la scultura a Bologna, 1267-1348, Bologne, 1982.

7. M. FouCAUlt, L'Ordre du discours, Paris, 1971, p. 17.

8. Cf. L VII 106 ou La fabrique de l'homme occidental, 1996, p. 18.

9. Corpus mysticum : l'Eucharistie et l'Église au Moyen Âge, Paris, 1944.

10. «La représentation et la notion d'efficience symbolique: un détour par la théorie des sacrements », Leçons III : Dieu au miroir, Paris, 1997, p. 199-215.

11. La parole efficace : signe, rituel, sacré, Paris, 2004.

12. Si l'on excepte le remarquable article d'A. Michel dans le t. 14 du Dictionnaire de théologie catholique.

13. Leçons IV : L'inestimable objet de la transmission, Paris, 1985, et sa suite : Le dossier occidental de la parenté, Paris, 1988.

14. A. Boureau a exposé très clairement ce que l'historien doit sur ce point aux recherches de

P. Legendre dans son compte rendu des Leçons IV, Médiévales 14, 1988, p. 134-137.

15. F. Noudelmann, Pour en finir avec la généalogie, Paris, 2004.

16. « Nietzsche, la généalogie, l'histoire », Dits et écrits, Paris, 1994, t. II, p. 154.

17. De Foucault cf. l'éd. récente du cours de 1981, Mal faire, dire vrai, Louvain, 2012.

18. P. LeGENDRE, «Qu'est-ce donc que la religion? ", Le Débat, 66, 1991, p. 42.

19. Annonce des conférences de Bruxelles, 1960, in J. LACAN, Le Triomphe de la religion, précédé de Discours aux catholiques, Paris, 2005, p. 13.

20. Cf. S. Benoit et ses fils, intro. et notes de Dom M.-F. Lacan, Paris, 1961 (dans la coll. « Textes pour l'histoire sacrée » de Daniel-Rops).

21. E. Roudinesco, Jacques Lacan : esquisse d'une vie, histoire d'un système de pensée, Paris, 1993.

22. A. DeSMAZIÈrEs, L'Inconscient au paradis, Paris, 2011, p. 196 ss.

23. P. Legendre a réfléchi, à partir de la thèse de Koyré sur Boehme, à l'écriture de l'histoire de la mystique (L III 165) : quid de La Fable mystique?

24. F. Dolto, L'Évangile au risque de la psychanalyse, t. I, Paris, $1980^{2}$, p. 87.

25. Car re-naître, "naître une seconde fois », c'est, en vocabulaire legendrien, être institué (L VI 49).

26. F. DOLTO, op. cit., p. 84 . 
INDEX

Mots-clés : droit canon, grégorianisme, liturgie, psychanalyse, ritualité, sacrement

\section{AUTEUR}

ALAIN RAUWEL

Université de Bourgogne 